\title{
Factores que modelan el patrón de minación de hojas en el arbusto Azara microphylla
}

\author{
Laila D. KaZimierski ${ }^{1} \&$ Alejandro G. Farji-Brener ${ }^{2, \bowtie}$ \\ ${ }^{1}$ Centro Atómico Bariloche, CONICET e Instituto Balseiro, S.C. de Bariloche, Argentina. ${ }^{2}$ Centro Regional Bariloche- \\ Universidad Nacional del Comahue y Laboratorio Ecotono, INIBIOMA-CONICET, Argentina.
}

\begin{abstract}
Resumen. La actividad de los herbívoros puede depender tanto de las características de los recursos como de las interacciones con otros organismos que dañan las hojas. Analizamos el efecto de la dureza de la hoja y la probabilidad de daño foliar sobre el recorrido y la eficiencia de las larvas del minador de hojas en el arbusto Azara microphylla. En comparación con el sector apical, el sector basal de las hojas fue más duro y mostró una probabilidad menor de daño foliar por herbívoros, hongos y/o patógenos. Los minadores tuvieron una actividad mayor en el sector basal, a pesar de que, en general, comenzaban su recorrido en el sector apical (que fue el sitio de mayor oviposición). La eficiencia resultó similar en ambos sectores foliares. Estos resultados sugieren que las interacciones con otros organismos que dañan las hojas cumplen un papel relevante en modelar la actividad forrajera de los minadores e ilustran cómo las construcciones que realizan los individuos pueden ser empleadas para estudiar su ecología y su comportamiento.
\end{abstract}

[Palabras clave: herbivoría, daño foliar, insectos minadores, Patagonia]

\begin{abstract}
AвSTRACT. Factors modeling the leaf miner pattern in the shrub Azara microphylla. The activity of herbivores may depend on resource characteristics as well as on interactions with other organisms that consume leaves. We analyzed the effect of leaf toughness and the probability of leaf damage on the activity and efficiency of leaf miners in the shrub Azara microphylla. Basal sectors of the leaves showed higher toughness and lower probability of damage by other herbivores, fungus and pathogens than apical leaf sectors. Leaf-miner activity was concentrated in basal leaf sectors, although they mainly initiated their activity in apical sectors (their preferred place to oviposit). Leaf-miner efficiency was similar in both leaf sectors. These results suggest that the interaction with other organisms that attack leaves play a relevant role modeling the foraging activity of leaf miners, illustrating how the construction that organisms build (i.e., trail miners) can be used to study their ecology and behavior.
\end{abstract}

[Keywords: herbivory, foliar damage, Patagonia, leaf-mining insects]

\section{INTRODUCCIÓN}

La actividad de los herbívoros depende de múltiples factores, tales comolas características de los recursos, el riesgo de depredación y las interacciones con diferentes tipos de consumidores primarios (Denno et al. 1995; Rzanny et al. 2012; McArthur et al. 2014). Por un lado, diversos estudios demuestran cómo la variación en la calidad nutricional y en los niveles de defensas de las hojas pueden determinar el nivel de daño foliar (Howard 1987; Coley \& Barone 1996). Por otro lado, la actividad de los herbívoros puede depender de la actividad de sus enemigos naturales (Marquis \& Whelan 1994; Böhm et al. 2011). Por último, el patrón de herbivoría también puede estar influido por la competencia intraespecífica con otros consumidores primarios (Denno et al. 1995; Fisher et al. 1999, 2000). En consecuencia, para comprender de manera integral los patrones de daño foliar es

Editor asociado: Diego Vázquez

$\triangle$ alefarij@yahoo.com aconsejable no solo analizar las características del recurso sino las interacciones entre los organismos que lo consumen.

Las interacciones indirectas entre diferentes tipos de consumidores primarios mediadas por la calidad del recurso juegan un papel relevante en la estructura de los ensambles de herbívoros (Denno et al. 1995). En particular, la actividad de un tipo de herbívoro puede afectar la actividad de otro tipo de herbívoro, propiciando relaciones positivas o negativas entre diferentes organismos del mismo gremio (Karban 1986; Kaplan \& Denno 2007). Por un lado, es lógico suponer que los herbívoros van a preferir atacar hojas o sectores de las hojas con menor nivel de defensas físico-químicas y mayor valor nutritivo. Ante este escenario, cierto nivel de daño foliar ocasionado por un herbívoro podría ser considerado como una señal de buena palatibilidad para otro organismo del mismo gremio. Además, los

Recibido: 23 de mayo de 2015, Fin de arbitraje: 13 de julio de 2015, Última versión revisada: 19 de septiembre, Aceptado: 26 de octubre de 2015 
daños causados por un tipo de herbívoro pueden debilitar a la planta atacada y hacerla más susceptible para otros organismos. En ambos casos se espera una asociación positiva entre el consumo foliar proveniente de diferentes organismos (Coley \& Barone 1996). Sin embargo, diferentes tipos de daño foliar pueden relacionarse de manera negativa si el daño inicial propicia la producción de defensas inducibles en la planta atacada, o si representa una reducción en la calidad o cantidad de recurso (i.e., competencia por explotación), o un incremento en el riesgo de mortalidad por depredación involuntaria (i.e., cuando un herbívoro consume una hoja que contiene larvas de otro herbívoro). En resumen, tanto la calidad del recurso como la interacción potencial entre los diferentes herbívoros pueden determinar el patrón de daño foliar.

Los minadores de hojas son un grupo interesante para analizar cómo los recursos y la competencia intraespecífica pueden modelar la actividad de los herbívoros. Estos insectos son larvas de Coleoptera, Diptera, Hymenoptera o Lepidoptera que viven en el interior del tejido de las hojas, del cual se alimentan. Los adultos depositan los huevos en las hojas. Al nacer, las larvas comienzan a moverse y consumen tejido del parénquima foliar; así es como forman un visible camino de minación. Cuando su desarrollo larval se completa, el insecto eclosiona de la pupa y deja la hoja (Sinclair \& Hughers 2012). Este modelo es un interesante sistema de estudio por varias razones. En primer lugar, las larvas poseen movilidad reducida y su actividad está restringida a una sola hoja, lo que facilita el seguimiento de su desarrollo completo. Además, es posible caracterizar diferentes sectores de una hoja por variaciones en sus niveles de calidad (e.g., dureza, concentración de nutrientes) y probabilidad de daño foliar por otros herbívoros, factores que pueden influir sobre la supervivencia y el desarrollo larval de los minadores (Bultman \& Faeth 1986). Por último, dado que la larva va creciendo a medida que se alimenta del parénquima foliar, se puede determinar con facilidad el sitio de inicio, la dirección de sus movimientos y el sitio de emergencia gracias al grosor del camino de minación (Sinclair \& Hughers 2012). Por todas estas razones, el camino que deja el minador durante su recorrido puede considerarse como su fenotipo extendido (Turner 2000) ya que dicha construcción es un reflejo de sus decisiones. En consecuencia, el simple análisis del camino de minación en una hoja puede servir de modelo para comprender mejor cómo los herbívoros lidian con las variaciones en los recursos y las interacciones con otros organismos de su mismo gremio.

Trabajos previos con minadores demostraron que sus recorridos en la hoja no son al azar, sino que están determinados por la calidad del recurso, el riesgo de depredación y la actividad de otros herbívoros (Denno et al. 1995; Fisher et al. 1999; Cornelissen \& Stiling 2008; Sinclair \& Hughers 2012). Por un lado, los minadores evaden los sectores de la lámina foliar donde forrajear requiere más esfuerzo (Bairstow et al. 2010; Sinclair \& Hughers 2012). Por otra parte, la supervivencia de los minadores depende de su eficiencia en evadir parasitoides, que son una de sus principales causas de mortalidad (Bultman \& Faeth 1986; Sinclair \& Hughers 2012). Sin embargo, la actividad de los minadores también puede verse muy afectada directa e indirectamente por la actividad de otros organismos degradadores o consumidores de hojas. Por ejemplo, la actividad de hongos y patógenos foliares, al igual que la presencia o la probabilidad de ocurrencia de daños ocasionados por insectos galígenos, raspadores o masticadores, reduce la cantidad y la calidad del recurso que los minadores pueden consumir (Bultman \& Faeth 1986; Fisher et al. 2000). Más aun, en algunos casos, la actividad de herbívoros masticadores puede ocasionar la muerte del minador si por casualidad es consumido junto con la hoja (Yamazaki \& Sugiura 2008). Finalmente, muchas hojas producen abscisión temprana ante altos niveles de daño foliar, lo que afecta de manera indirecta la supervivencia del minador. Pese a la importancia potencial del efecto de otros herbívoros y/o patógenos sobre los patrones de minación, existen pocos estudios que analizan su influencia (Yamazaki \& Sugiura 2008; Fisher et al. 1999, 2000), y ninguno en bosques templados del hemisferio sur.

Nosotros determinamos, de forma indirecta, la importancia de la calidad del recurso y la actividad de otros herbívoros sobrelos patrones de minación analizando los recorridos de los minadores en hojas de Azara microphylla, un arbusto del sotobosque andino-patagónico. Para ello, clasificamos diferentes sectores de la hoja según su dureza y la probabilidad de sufrir daño foliar, y determinamos los sectores de mayor recorrido del minador y aquellos donde su crecimiento es más eficiente. 


\section{MATERIALES y MÉTODOS}

\section{Sitio de estudio}

El sitio de estudio se encuentra en el Parque Municipal Llao-Llao, $30 \mathrm{~km}$ al oeste de la ciudad de San Carlos de Bariloche, Argentina (41 ${ }^{\circ} 8^{\prime}$ S, $71^{\circ} 19^{\prime}$ O). La precipitación anual es de $\sim 1800 \mathrm{~mm}$ y la temperatura promedio varía entre $2^{\circ} \mathrm{C}$ en invierno y $13^{\circ} \mathrm{C}$ en verano. El área de estudio es un bosque mixto dominado por los árboles Nothofagus dombeyi y Austrocedrus chilensis, en donde el arbusto Azara microphylla forma parte del sotobosque y presenta una abundancia variable. Sus hojas son pequeñas, $\sim 2 \mathrm{~cm}$ de longitud (Figura 1), y de color verde oscuro.

\section{Metodología general}

Para analizar el efecto de la dureza y los niveles de daño foliar sobre los patrones de minación en Azara microphylla estimamos en las hojas: a) su dureza, b) la frecuencia de todos los tipos de daño, c) la ubicación y la intensidad de daños diferentes a la minación, para calcular una probabilidad de daño foliar, d) la ocurrencia de minadores en los diferentes sectores de la hoja, describiendo el sitio de inicio y final del camino de minación, y e) la eficiencia del minador. En todas las mediciones las hojas fueron divididas en dos sectores de igual área: basal y apical (Figura 1). A continuación detallamos cada medición.

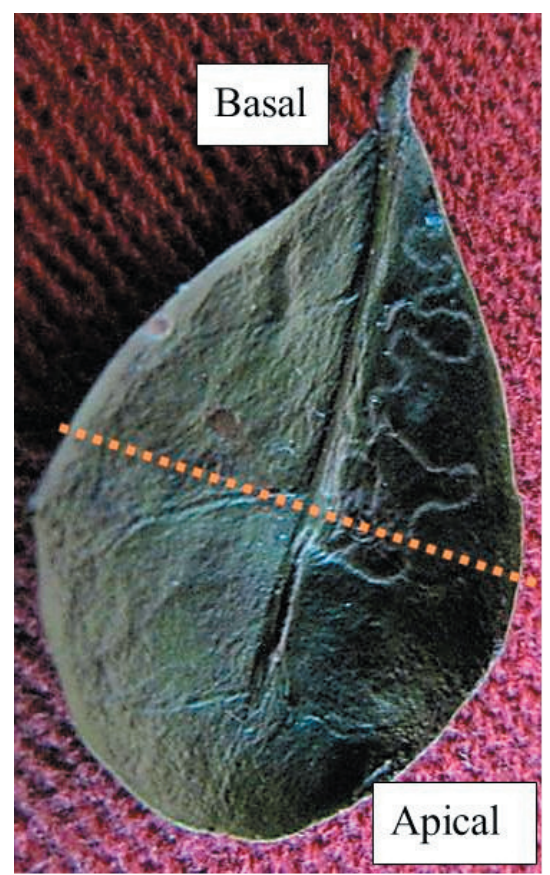

Figura 1. Hoja minada de Azara microphylla $(\sim 2 \mathrm{~cm})$ dividida en los sectores basal y apical que fueron empleados en el muestreo.

Figure 1. Mined leaf of Azara microphylla $(\sim 2 \mathrm{~cm})$ divided in basal and apical sectors as were used in the sampling design.

\section{Dureza de hoja}

Se midió la dureza de cada sector foliar en 52 hojas sin daños ni signos de minación, provenientes de 6 árboles. Para ello se empleó un penetrómetro foliar marca Pesola $\AA$, que permite determinar la fuerza necesaria para romper la lámina foliar. Se realizó una medición en cada sector (apical y basal) de cada hoja, evitando las nervaduras. Las estimaciones de dureza entre ambos sectores fueron comparadas empleando una prueba de t-pareada.

\section{Frecuencia de los diferentes tipos de daño foliar}

Para determinar la frecuencia de los diferentes tipos de daño foliar recolectamos 300 hojas al azar, provenientes de 8 árboles. La elección de las hojas se realizó de forma estratificada para que todas las secciones de la copa del arbusto (e.g., basal, media y terminal, externas e internas) estuvieran representadas en la muestra. Cada hoja fue asignada a sólo una de estas cuatro categorías: con sólo minación, con daños foliares pero sin minación, con ambos tipo de daño, y sin daño. La frecuencia de cada categoría fue expresada como

$$
\text { Frecuencia }(\%)=\left(\mathrm{N}_{\mathrm{i}} / \mathrm{N}_{\mathrm{T}}\right)^{*} 100
$$

donde $\mathrm{N}_{\mathrm{i}}$ es el número de hojas de la categoría i y $\mathrm{N}_{\mathrm{T}}$ es el total de hojas analizadas (en este caso, 300). En las hojas con ambos tipos de daño (minación y otro), determinamos la existencia de coincidencia espacial y su ubicación (basal o apical).

\section{Daños foliares diferentes a la minación: ubicación e intensidad}

Para estimar la probabilidad de daño foliar recolectamos un total de 292 hojas maduras sin signos de minación, pero con evidencia de daño foliar, provenientes de 13 árboles elegidos al azar ( 25 hojas/arbusto). Las hojas fueron elegidas de forma estratificada, para que todas las secciones de la copa del arbusto (e.g., basal, media y terminal, externas e internas) estuvieran representadas en la muestra. Consideramos la pérdida de lámina foliar, presencia de agallas y signos producidos por hongos o patógenos como daño foliar, ya que pueden potencialmente causar la muerte, dañar la larva del minador y/o limitar la calidad/cantidad de su alimentación. Para estimar la probabilidad de daño foliar integramos en una única medida la probabilidad de ocurrencia y la intensidad relativa de daño foliar para cada sector de la hoja. La probabilidad de ocurrencia de daño fue estimada contabilizando cuántas de las 292 hojas presentaban daño en su parte basal o apical (por lo general, el daño se encontraba localizado en uno $\mathrm{u}$ otro sector). Para calcular la intensidad de daño foliar cuantificamos de manera visual el porcentaje de daño en cada sector de la hoja utilizando las siguientes categorías: $0=0 \%, 1=1-6 \%, 2=6-12 \%, 3=12$ $25 \%, 4=25-50 \%, 5=50-100 \%$. Con estas categorías calculamos un índice de herbivoría (IH) para cada sector de la hoja (basal y apical), como: 


$$
\mathrm{IH}=\sum_{\mathrm{i}}\left(\mathrm{n}_{\mathrm{i}}{ }^{*} \mathrm{i}\right) / \mathrm{N}_{\mathrm{T}}
$$

donde $n_{i}$ es la frecuencia de hojas por categoría, $i$ representa la categoría del daño y $\mathrm{N}_{\mathrm{T}}$ es el total de hojas. Al IH lo expresamos como porcentaje foliar dañado. Este índice es considerado un estimador adecuado de daño foliar y ha sido muy utilizado en diversos trabajos y hábitats (Domínguez et al. 1989; Dirzo \& Domínguez 1995; Cuautle \& RicoGray 2003). Con esta información estimamos la probabilidad de daño foliar para cada sector de la hoja (basal y apical) $\mathrm{P}_{\mathrm{df}}$ como:

$$
\mathrm{P}_{\mathrm{df}}=\left(\mathrm{N}_{\mathrm{S}} / \mathrm{N}_{\mathrm{T}}\right) *\left(\mathrm{IH} / \mathrm{IH}_{\max }\right)
$$

donde $\mathrm{N}_{\mathrm{S}}$ es la frecuencia con que una sección sufre daño, $\mathrm{N}_{\mathrm{T}}$ es el índice de herbivoría del sector, $\mathrm{e}$ $\mathrm{IH}_{\text {max }}=5$ (máximo nivel posible de daño foliar). De esta forma, la probabilidad de daño foliar de cada sector de la hoja integró tanto la frecuencia con que sufren herbivoría como su intensidad. Los valores de cada sección fueron comparados mediante una prueba de diferencia de proporciones.

\section{Patrones de minación: ubicación, recorrido y eficiencia}

La frecuencia de minación en cada sector de la hoja fue realizado en una muestra de 126 hojas minadas (sin otro tipo de daño), provenientes de 8 árboles. Cada hoja fue caracterizada con minación en la base o en su ápice en los casos en que al menos $75 \%$ del recorrido del minador se encontrara en dicho sector. La proporción de hojas minadas en la base o en el ápice fueron comparadas entre árboles mediante una prueba de $X^{2}$. Dado que esta relación fue consistente entre árboles (ver resultados), se comparó la proporción de hojas minadas en la base y ápice mediante una prueba de proporciones con la totalidad de las hojas. Las frecuencias esperadas fueron consideradas de un $50 \%$ para cada sector, ya que cada uno representaba la mitad del área de la hoja.

Para determinar los sitios de inicio y fin del recorrido del minador empleamos 285 hojas de 13 árboles. Como fue explicado anteriormente, dado que a medida que la larva consume parénquima va creciendo, el incremento del grosor del camino indica su recorrido (ver Figura 1). En consecuencia, para cada hoja determinamos si el inicio y final de su recorrido ocurría en la parte basal o apical. Todos los recorridos medidos concluían con la eclosión de la larva. Las pocas hojas con señales de recorridos incompletos o con signos de haber sido parasitadas fueron descartadas del análisis. La probabilidad de cambio (i.e., que un camino iniciado en la base o en el ápice termine en el ápice o en la base, respectivamente) fue analizado con una prueba de proporciones de McNemar para datos dependientes (Zar 1999).

Estimamos la eficiencia del minador en una submuestra de 123 hojas provenientes de 8 árboles. En cada hoja medimos la distancia recorrida (en $\mathrm{cm}$ ) de toda la trayectoria del minador y el ancho del sector de origen y final en cada caso. Además, determinamos en qué sector de la hoja (basal o apical) se encontraba más del $75 \%$ del recorrido total. Estas mediciones fueron realizadas en fotografías de las hojas empleando el programa Image $®$. Dado que el minador va alimentándose y creciendo a medida que avanza por el parénquima foliar, estimamos la eficiencia relativa de forrajeo del minador $\left(\mathrm{E}_{\mathrm{r}}\right)$ como

$$
E_{r}=\left(G_{f}-G_{i}\right) / d
$$

donde $G_{f}$ y $G_{i}$ representan el grosor final e inicial del sendero de minación, respectivamente, y d, la distancia total recorrida. La diferencia $\left(G_{f}-G_{i}\right)$ da cuenta de cuánto se alimenta la larva, mientras que d representa cuánto debió moverse para obtener ese alimento. En consecuencia, valores crecientes de Er representan mayor/mejor tasa de asimilación de alimento por distancia recorrida, o sea, mayor eficiencia relativa de forrajeo. Las eficiencias entre los sectores de las hojas fueron comparadas mediante un análisis de varianza anidado, en el cual los sectores (i.e., hojas) fueron anidados a los árboles.

\section{RESUltados}

\section{Dureza de hoja}

El sector basal de la hoja presentó mayor dureza que el sector apical. Mientras que para romper la lámina foliar en el sector basal fue necesario aplicar una fuerza de $70 \pm 10 \mathrm{~g}$ (media $\pm \mathrm{ES})$, en el sector apical sólo fue necesario aplicar $47 \pm 5 \mathrm{~g}$ (t-pareada $=3.05$, $P=0.04$; Figura 2a).

\section{Frecuencia de los diferentes tipos de daño foliar}

De las 300 hojas muestreadas al azar, 192 presentaron algún tipo de daño (64\%). Si se consideran todas las hojas (con y sin daño), $26 \%(n=79)$ presentó sólo minadores, $28 \%$ $(\mathrm{n}=84)$ algún tipo de daño foliar sin rastros de minación, y 10\% (n=29) ambos tipos de daño. En consecuencia, 36\% de las hojas muestreadas presentaron minación $(n=79+29)$ y 38\% daños foliares diferentes a la minación $(\mathrm{n}=84+29)$. Si se consideran sólo las 108 hojas minadas (79+29), 27\% sufrió daño foliar por otros organismos (29/108). Cuando las hojas poseían ambos tipos de daño, éstos coincidían mayormente en la parte apical $\left(X^{2}=12\right.$, g.l. $=2$, $P=0.02$; Figura 2b). En el $66 \%$ de los casos, el recorrido del minador y otros tipos de daños foliares coincidían en el ápice de la hoja, mientras en el sector basal sólo coincidían en el 15\%. No coincidieron en 19\% de los casos. 

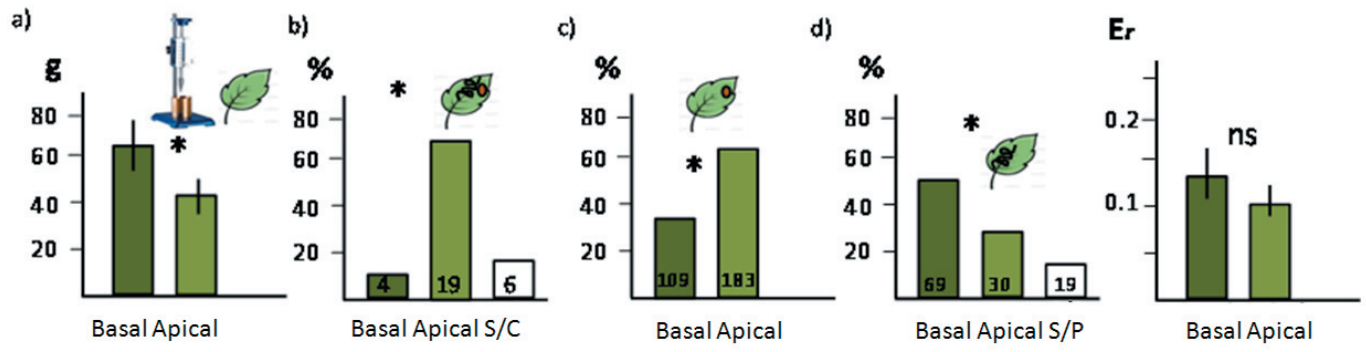

Figura 2. Resultados de las mediciones de: a) dureza de hoja, b) cantidad de hojas en donde ambos tipos de daño (minadores vs. otros) coincidían espacialmente en los diferentes sectores ( $\mathrm{S} / \mathrm{C}=\sin$ coincidencia), c) cantidad de hojas en las cuales los daños foliares (excluyendo minadores) eran más frecuentes en los sectores basales o apicales de la hoja, d) cantidad de hojas en las cuales la minación era más frecuente en los sectores basales o apicales de la hoja, y e) eficiencia relativa del minador $\left(\mathrm{E}_{\mathrm{r}}=\right.$ incremento del grosor del camino ponderado por la distancia recorrida). Todos los resultados son expresados en porcentajes, excepto la dureza de hoja que es expresada en gramos necesarios para su perforación y la eficiencia (ver métodos). Los valores dentro de las barras son las frecuencias absolutas (e.g., número de hojas en cada categoría). Los asteriscos indican diferencias estadísticamente significativas; ns = no significativas.

Figure 2. Results of measurements of: a) leaf toughness, b) number of leaves where both types of foliar damages (miner vs. others) spatially overlapped in the different leaf sectors (S/C = no overlap), c) number of leaves where foliar damage (excluding miners) were more frequent in basal or apical leaf sectors, $\mathrm{d}$ ) number of leaves where miner was more frequent in basal or apical leaf sectors, and e) efficiency ( $E_{r}=$ measured as trail width increment/distance). Leaf toughness was expressed as grams needed to perforate the leaf (see methods). The rest of results were expressed in percentage. Values inside the bars are absolute frequencies (e.g., number of leaves in each category). Asterisks imply statistical significant difference; $n s$ = statistical non-significant differences.

Daños foliares diferentes a la minación: ubicación e intensidad

Las hojas de Azara microphylla presentaron una mayor frecuencia de daño foliar en el sector apical de la hoja que en el basal. De las 292 hojas medidas, 183 (63\%) presentaron daño foliar en el sector apical, mientras que 109 (37\%) evidenciaron daños foliares en el sector basal $\left(X^{2}=18.7\right.$, g.l. $=1, P<0.001$; Figura 2c). Este patrón de mayor daño en el sector apical fue consistente entre los diferentes árboles $\left(X^{2}=9.4\right.$, g.l. $=1, P=0.23)$. Además, la intensidad de daño presentó una fuerte tendencia a ser mayor en la parte apical de la hoja. Mientras que el nivel de herbivoría en la parte basal fue alrededor del $10 \%(\mathrm{IH}=2.5 \pm 0.15)$, la parte apical de la hoja sufrió alrededor de un $20 \%$ de daño $(\mathrm{IH}=3.0 \pm 0.15$ [media $\pm \mathrm{ES}$ ], $\mathrm{t}=1.9, P=0.06)$. En consecuencia, la probabilidad de daño foliar, $\mathrm{P}_{\mathrm{d}^{\prime}}$ fue significativamente mayor en la parte apical de la hoja comparada con la parte basal ( 0.37 versus 0.19 , respectivamente, $P=0.001$, prueba de diferencia de proporciones).

\section{Patrones de minación: ubicación, recorrido y eficiencia}

La minación se ubicó preferencialmente en el sector basal de las hojas $\left(X^{2}=21.6\right.$, g.l. $=2$, $P<=0.001)$. De las 126 hojas, en 69 (55\%) los minadores se ubicaron en la parte basal, en 38 en el sector apical (30\%), y en $19(15 \%)$ no se pudo determinar una preferencia por algún sector. Esta mayor ocurrencia de minación en el sector basal de las hojas fue consistente entre los diferentes árboles $\left(X^{2}=6.7\right.$, g.l. $=2, P=0.46$; Figura 2d). Respecto a los sitios de comienzo y fin de las caminatas, en su gran mayoría, el inicio del recorrido ocurrió en el ápice (en 159 casos de $285[56 \%], X^{2}=3.8$, g. $\left.1 .=1, P=0.05\right)$, pero terminó principalmente en la base (en 186 casos de 285). Esta preferencia por dirigirse hacia la base fue estadísticamente significativa $\left(X^{2}=58.4\right.$, g.l. $=1, P<0.001$; prueba de McNemar). Mientras que $80 \%$ de los minadores que iniciaron su recorrido en la base terminaron su

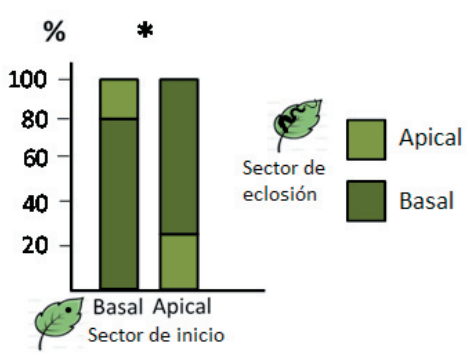

Figura 3. Frecuencia de cambios entre los sitios de inicio y fin del recorrido de los minadores. Las categorías del eje $\mathrm{X}$ representan el sector de inicio del recorrido del minador (i.e., donde la hembra puso el huevo), y los colores de las barras el sector de eclosión de la larva (i.e., el final del recorrido). El asterisco significa diferencias estadísticamente significativas. El sector basal es el preferido para forrajear y eclosionar.

Figure 3. Changes between the start and end point of the miner trail. The categories in the $\mathrm{X}$ axis represent the start trail point (i.e., the site where the insect deposit her egg), and the different colors inside the bar the leaf sector when the larva emerged (i.e., the end of the miner trail). The asterisk means statistical significant differences. The basal sector is preferred to forage and emerge 
recorrido en el mismo sector (100 de 126 casos), sólo $25 \%$ de los que iniciaron su recorrido en el ápice (40 de 159 casos) lo terminaron en ese sector (Figura 3). De las 123 hojas empleadas para medir la eficiencia, en el $80 \%(n=99)$ la minación se encontraba agrupada en uno de los sectores ( 37 en el ápice y 62 en la base). En el resto de las hojas $(n=24)$ los recorridos se encontraban en ambos sectores, por lo cual estos casos no fueron incluidos en el análisis. Considerando solo las hojas en las cuales los recorridos claramente se ubicaban en la base o el ápice, la eficiencia del minador fue similar entre ambos sectores (Figura 2d). Mientras que la eficiencia fue de $0.13 \pm 0.02$ en la base, en el ápice alcanzó valores de $0.10 \pm 0.01\left(\mathrm{~F}_{8,86}=1.2\right.$, $P=0.24)$. Este patrón fue consistente en todos los árboles $\left(\mathrm{F}_{7,86}=4.3, P<0.25\right)$.

\section{DisCUSIÓN}

Los sectores basal y apical de las hojas de Azara microphylla presentaron diferentes niveles de dureza, de probabilidad de daño foliar y de actividad de minadores. El sector apical fue $70 \%$ más blando que el sector basal y sufrió el doble de intensidad de daño foliar por organismos diferentes a los minadores. La actividad de los minadores se concentró en el sector basal, donde fue $60 \%$ más frecuente y donde eclosionaron el $80 \%$ de las veces, pese a que mayoritariamente comenzaban su recorrido en el sector apical. La eficiencia de forrajeo de los minadores fue similar en ambos sectores de la hoja. Todos estos resultados sugieren que la mayor actividad de los minadores de Azara microphylla en el sector basal de la hoja se asocia más a la actividad de otros organismos que dañan el tejido foliar que a las características de la hoja, lo cual apoya la idea de que las interacciones interespecíficas cumplen un papel relevante en afectar los patrones de actividad y niveles de daño foliar (Denno et al. 1995; Fisher et al. 1999, 2000).

Nuestros resultados son consistentes con el patrón general de que las hojas son más duras en las partes basales que en las apicales (Choong 1996; Lucas et al. 2000; Teaford et al. 2006), y con que la dureza foliar limita el consumo de los herbívoros (Coley \& Barone 1996). En este estudio, la mayoría de los recorridos de los minadores comenzaron en el sector apical de las hojas; esto sugiere que los adultos de los minadores eligen los sectores más blandos de la hoja para oviponer. Sin embargo, la mayor parte de los recorridos y el sitio de eclosión sucedieron en el sector basal. Estos resultados apoyan la idea que postula posibles intereses contrapuestos entre el adulto y su descendencia, debido a que los mejores sitios para oviponer no necesariamente son los mejores para el desarrollo y crecimiento larval (Thompson 1988 y referencias allí citadas).

Diversas evidencias sugieren que en el arbusto Azara microphylla la actividad de los minadores está modelada más por su interacción con otros organismos que producen daño foliar que por características de las hojas. Por un lado, los minadores concentraron su recorrido en los sectores más duros de las hojas, resultado opuesto al esperado si la dureza foliar funcionase como una defensa contra estos organismos. Segundo, la eficiencia de forrajeo de los minadores fue similar en ambos sectores de la hoja, lo que sugiere que ambos sectores de la hoja son igualmente nutritivos. No obstante, dado que en este trabajo no medimos concentración de defensas químicas ni nutrientes foliares, esta hipótesis necesita de más mediciones para sustentarla. Por otra parte, nuestros resultados sobre los patrones de daño foliar y actividad de los minadores sugieren una interacción negativa intragremial. Mientras los daños foliares ocasionados por otros organismos se concentraron en el sector apical, la actividad de los minadores se concentró en el basal. En adición, pese a que los recorridos de forrajeo comenzaron generalmente en el sector apical, la mayoría fueron hallados en el sector basal de la hoja, lugar donde se produjo la mayor eclosión. Por último, un gran porcentaje de hojas minadas $(\sim 30 \%)$ resultó dañado por otros organismos y, cuando eso sucedía, la superposición entre el recorrido del minador y el daño ocurría principalmente en la parte apical. Todas estas evidencias sugieren que la concentración espacial de la actividad de los minadores en la parte basal es un mecanismo que reduce la probabilidad de encuentro con otros organismos que dañan las hojas, evadiendo de esta forma alimentarse de partes poco nutritivas (atacadas por hongos, patógenos o galígenos) o exponerse al riesgo de ser consumido casualmente por otro herbívoro.

El mecanismo de selección por el cual los minadores concentran su actividad de alimentación en un sector de la hoja es aún poco conocido. Una posibilidad es un tropismo negativo ante encuentros reiterados con las partes foliares dañadas. Sin embargo, un comportamiento innato de preferencia por 
el sector basal sería deseable para mejorar su desempeño y reducir riesgos de muerte por consumo accidental. En este sentido, es poco probable que las larvas de minadores puedan predecir y/o tener información previa de los sectores de las hojas con más riesgo de sufrir daño, dado que pasan toda su vida larval en una misma hoja y su percepción del mundo exterior es limitada. No obstante, características intrínsecas de la hoja como variaciones en los patrones de nervaduras o cambios en el parénquima foliar podrían ser señales asociadas a sectores con mayor o menor riesgo de daño que las larvas pueden percibir directamente. Nuestros resultados sugieren que la dureza foliar podría ser una de esas señales, ya que normalmente la dureza se asocia de forma negativa con la probabilidad de daño foliar (Coley \& Barone 1996). Los minadores minimizarían la probabilidad de encontrarse con otros herbívoros al concentrar la actividad forrajera en las partes más duras de las hojas; esto reduciría las consecuencias de la competencia por explotación.

La mayor densidad de recorrido en el sector basal de las hojas también podría explicarse por otros motivos diferentes a la hipótesis de interferencia negativa con otros organismos. Por ejemplo, los minadores podrían preferir forrajear en la parte basal de las hojas porque allí el parénquima foliar recibe nutrientes de forma más directa o para evadir sectores de mayor densidad de nervaduras. Sin embargo, no está claro que los sectores basales de las hojas sean más nutritivos que los apicales, y en el arbusto estudiado no hay un patrón definido de nervaduras más densas en sectores apicales. Esto hace poco probable las hipótesis alternativas mencionadas.

En resumen, los minadores realizan la mayor parte de su desarrollo como larva en el sector basal de las hojas de Azara microphylla, que es un sitio con menor riesgo de daño foliar y mayor dureza que el sector apical. Estos resultados resaltan la importancia de las interacciones interespecíficas y la competencia por explotación como posibles modeladores de la actividad de los herbívoros, y cuestionan la dureza de hoja como una característica foliar que limita el desempeño de las larvas de los minadores. Finalmente, este trabajo es un ejemplo de cómo las construcciones que realizan los organismos (en este caso, los senderos de minación) pueden emplearse para estudiar el comportamiento animal. Esto enfatiza la utilidad del concepto de fenotipo extendido en el estudio de las interacciones bióticas.

Agradecimientos. Este trabajo fue realizado en sus inicios como el proyecto final de la materia "Ecología de las Interacciones Planta-Animal" de la carrera de Biología del Centro Regional de la Universidad del Comahue, Bariloche, Argentina. Agradecemos a todos los participantes del año 2014, quienes colaboraron discutiendo la idea piloto. Agradecemos también a Damián Preisz por su ayuda con el trabajo de campo y las fotografías de las hojas analizadas. Dos revisores anónimos realizaron valiosas sugerencias que mejoraron notablemente el trabajo.

\section{BiBLIOGRAFÍA}

Bairstow, K; K Clarke; M McGeoch \& N AndRew. 2010. Leaf miner and plant galler species richness on Acacia: relative importance of plant traits and climate Oecologia, 163:437-448

BöHM, SM; K Wells \& EKV KaLKO. 2011. Top-Down Control of Herbivory by Birds and Bats in the Canopy of Temperate Broad-Leaved Oaks (Quercus robur). PLOS ONE, 6:e17857. doi:10.1371/journal.pone.0017857.

BultMAN, T \& S FAETH. 1986. Effect of within-leaf density and leaf size on pupal weight of a leaf-miner, cameraria (lepidoptera: gracillariidae). The Southwestern Naturalist, 31: 201-206.

CHOONG, MF. 1996. What makes a leaf tough and how this affects the pattern of Castanopsis fissa leaf consumption by caterpillars. Functional Ecology, 10:668-674.

Coley, PD \& JA Barone. 1996. Herbivory and plant defenses in tropical forests. Annual Review of Ecology and Systematics, 27:305-335.

Cornelissen, T \& P Stiling. 2008. Clumped distribution of oak leaf miners between and within plants. Basic and Applied Ecology, 9:67-77.

Cuautle, M \& V Rico-Gray. 2003. The effect of wasps and ants on the reproductive success of the extrafloral nectaried plant Turnera ulmifolia (Turneraceae). Functional Ecology, 17:417-423.

DenNo, RF; MS McCluRe \& JR OtT. 1995. Interspecific interactions in phytophagous insects: competition reexamined and resurrected. Annual Review of Entomology, 40:297-331.

Dirzo, R \& C DomínguEz. 1995. Plant-herbivore interactions in Mesoamerican tropical dry forests. Pp. 304-345 in: Bullock, S; S Mooney \& E Medina (eds.). Seasonally dry tropical forests. Cambridge University, Massachusetts.

Domínguez, CA; R Dirzo \& SH Bullock. 1989. On the function of floral nectar in Croton suberosus (Euphorbiaceae). Oikos, 56:109-114.

Fisher; A; SE Hartley \& M Young. 1999. Behavioural responses of the leaf-chewing guild to the presence of Eriocrania mines on silver birch (Betula pendula). Ecological Entomology, 24:15-162

Fisher; A; SE Hartley \& M Young. 2000. Direct and indirect competitive efects of foliage feeding guilds on the performance of the birch leaf-miner Eriocrania. Journal of Animal

Ecology, 69:165-176.

HowARD, JJ. 1987. Leaf-cutting and Diet Selection: Relative Influence of Leaf Chemistry and Physical Features. Ecology, 69:250-260. 
KAPLAN, I \& RF DENNO. 2007. Interspecific interactions in phytophagous insects revisited: a quantitative assessment of competition theory. Ecology Letters, 10: 977-994.

KARBAN, R. 1986 Interspecific competition between folivorous insects on Erigeron glaucus. Ecology, 67: 1063-072.

LuCAs, PW; IM Turner; NJ DOMiny \& N Yamashita. 2000 Mechanical defenses to herbivory. Ann. Bot., 86:913920.

Marquis, RJ \& CJ Whelan. 1994. Insectivorous birds increase growth of white oak through consumption of leaf-chewing insects. Ecology, 75:2007-2014.

McArthur, C; P Banks; P Boonstra; R JenNiF \& J Forbey. 2014. The dilemma of foraging herbivores: dealing with food and fear. Oecologia, 176:677-689.

Rzanny, M; A KuU \& W VoIgt. 2012. Bottom-up and topdown forces structuring consumer communities in an experimental grassland. Oikos, 122:967-976.
SinCLAIR, R \& L Hughers. 2012. Leaf miners: The hidden herbivores. Austral Ecology, 35:300-313.

Teaford, MF; PW Lucas; PS Ungar \& KE Glander. 2006. Mechanical Defenses in Leaves Eaten by Costa Rican Howling Monkeys (Alouatta palliata). American Journal of Physical Anthropology, 129:99-104.

THOMPSON, J. 1998. Evolutionary ecology of the relationship between oviposition reference and performance of offspring in phytophagous insects. Entomologia Experimentalis et Applicata, 47:3-14.

TURnER, JS. 2000. The Extended Organism: The Physiology of Animal-Built Structures. Harvard University Press, Cambridge, Massachusetts.

YAMAZAKI, K \& S SugIURA. 2008. Deer predation on leaf miners via leaf abscission. Naturwissenschaften, 95: 263-268.

Zar, JH. 1999. Biostatistical Analysis. Prentice-Hall. London. Pp. 717. 